fähigkeits-Glow-Kurve ist in Abb. 2 als Kurve I wiedergegeben (Saugspannung $10 \mathrm{~V}$ ). Sie war gut reproduzierbar.

Wird der beschriebene Versuch wiederholt, indem jedoch lediglich in der (gleichlangen) Dunkelpause für die Dauer von 1 min eine Wechselspannung $(50 \mathrm{~Hz})$

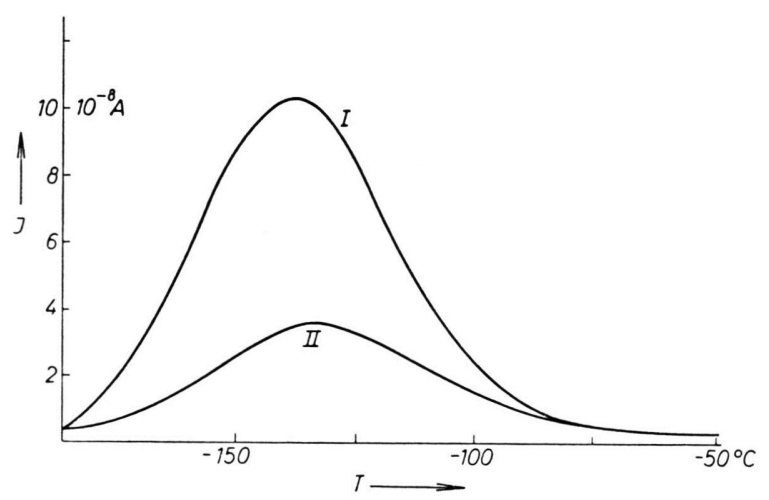

Abb. 2.

* Eine solche Anregung braucht sich im allgemeinen keineswegs homogen auf das Dielektrikum zu erstrecken, wie durch Untersuchung des Feldverlaufs festgestellt wurde ${ }^{5,6}$. von $2 \mathrm{kV}$ an die Elektroden $\mathrm{K}_{1}$ und $\mathrm{K}_{2}$ gelegt wird, die anschließend während 5 min kontinuierlich auf $0 \mathrm{~V}$ heruntergeregelt wird (während dieser Zeit waren die Schalter $S_{1}$ und $S_{2}$ geöffnet), so ergibt sich jetzt die Leitfähigkeits-Glow-Kurve II (Abb. 2).

Diese Glow-Kurve liegt beträchtlich unter der Kurve I und zeigt damit an, daß die Zahl der in den Hafttermen gefangenen Elektronen vor Aufnahme der Kurve II deutlich kleiner als vor Kurve I war. Eine entsprechende Verminderung der Haftelektronenkonzentration muß durch Wirkung der hohen Wechselfelder zustande gekommen sein, was in einfacher Weise nur durch eine elektrische Anregung von Elektronen aus Hafttermen in das Leitungsband und anschließende Rekombination erklärt werden kann.

Damit haben wir ganz entsprechende Ergebnisse, wie wir sie früher ${ }^{3,4}$ bei direkter Kontaktierung erhalten haben, jetzt auch bei Feldzuführung über isolierende Zwischenschichten gewonnen, wodurch eine Beeinflussung der Meßergebnisse durch eine Injektion von Stromträgern ausgeschlossen wurde.

Unsere bisherige Beschreibung der Experimente durch eine Feldanregung wird dadurch bestätigt * .

5 K. W. Böer u. U. Kümmel, Ann. Phys., Lpz. 20, 303 [1957].

${ }^{6}$ K.W. Böer u. U. Kümmel, Z. Naturforschg. 12 a, 667 [1957]; Ann. Phys., Lpz., im Druck.

\section{Die Gestalt des Leitfähigkeitsbandes von Indiumarsenid}

\author{
Von D. GEIsT
}

2. Physikalisches Institut der Universität Köln

(Z. Naturforschg. 13 a, 699-700 [1958] ; eingegangen am 3. Juli 1958)

In Halbleitern mit kleiner effektiver Masse am Bandrand rückt die Fermi-Energie bei mäßigen und hohen Trägerkonzentrationen verhältnismäßig weit ins Band vor. Dann werden Zustände besetzt, für die häufig keineswegs mehr der einfache Zusammenhang $E=\hbar^{2} k^{2} / 2 m^{*}$ zwischen Energie $E$ und Betrag des Ausbreitungsvektors $k$ mit konstanter effektiver Masse $m^{*}$ besteht. Man kann diese Gleichung formal beibehalten, wenn man in $E=\hbar^{2} k^{2} / 2 m_{1} ; \mathrm{d} E / \mathrm{d} k=\hbar^{2} k / m_{2} ; \mathrm{d}^{2} E / \mathrm{d} k^{2}=\hbar^{2} / m_{3}$

die Größen $m_{1}, m_{2}$ und $m_{3}$ als voneinander verschieden und als Funktionen der Energie betrachtet. Die Energie hängt dann nicht mehr quadratisch von $k \mathrm{ab}$, doch sind die Flächen konstanter Energie im E-Raum immer noch Kugeln.

Führt man die Relationen (1) in die allgemeinen Formeln für die magnetische Suszeptibilität quasifreier Träger ein ${ }^{1}$, so erhält man im Gültigkeitsbereich der Fermi-Statistik für den temperaturunabhängigen Term ${ }^{2}$

1 A. H. W ILson, The Theory of Metals, 2. Aufl., University Press, Cambridge 1954, p. 175. - H. FröHLich, Elektronentheorie der Metalle, Springer-Verlag, Berlin 1936, S. 145.

2 Auf eine ausführliche Ableitung wird hier verzichtet.

$$
\varkappa=\frac{3^{1 / 3}}{\pi^{4 / 3}} \mu_{B}^{2} \frac{m_{1}}{\hbar^{2}} n^{1 / 3}\left\{1-\frac{1}{9} \frac{m}{m_{1}}\left(\frac{2 m}{m_{3}}+\frac{m}{m_{2}}\right)\right\} .
$$

$m_{1}, m_{2}$ und $m_{3}$ sind an der Stelle $E=\zeta$ (Fermi-Energie) zu nehmen. ( $\mu_{\mathrm{B}}$ BoHr-Magneton, $m$ Elektronenmasse, $2 \pi \hbar$ Plancksches Wirkungsquantum).

Für Indiumarsenid ist ein entsprechender Vergleich zwischen experimentellen Suszeptibilitätsmessungen und theoretischen Berechnungen der Bandstruktur möglich.

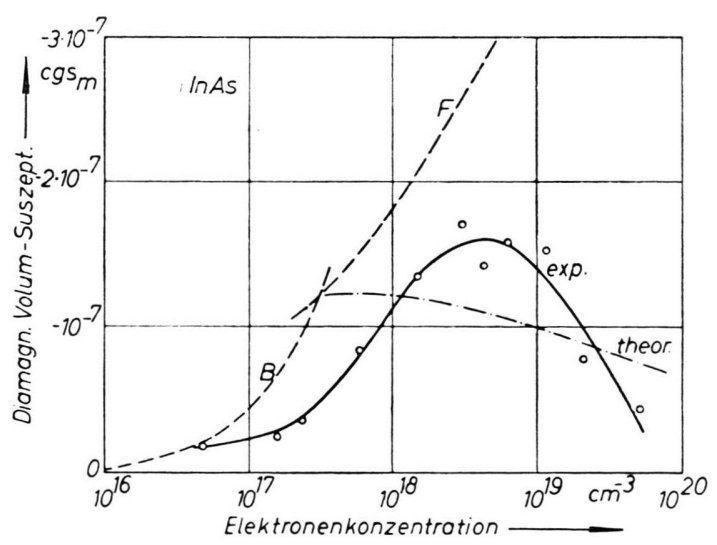

Abb. 1. Magnetische Volumsuszeptibilität der Elektronen in Indiumarsenid. Exp.: Meßwerte ${ }^{4}$; theor.: berechnet nach Formel (2); $B$ und $F$ : berechnet für konstante effektive Masse $\left(m^{*} / m=0,04\right)$. 
Stern hat nach der $(\mathfrak{p} \cdot \mathfrak{k})$-Approximation berechnete Werte von $m_{1}, m_{2}$ und $m_{3}$ für Elektronenkonzentrationen zwischen $3 \cdot 10^{16}$ und $3 \cdot 10^{19} \mathrm{~cm}^{-3}$ angegeben ${ }^{3}$, aus denen sich nach (2) die zu erwartende Suszeptibilität berechnen läßt. Einen Vergleich mit den kürzlich mitgeteilten ${ }^{4}$ Messungen gibt Abb. 1. Während sich für konstante effektive Masse ein steiler Anstieg der Suszeptibilität mit der Trägerkonzentration ergeben müßte [gestrichelte Kurven für $m^{*} / m=0,04$ für BoltzmanNbzw. Fermi-Statistik ( $B$ bzw. $F$ ) ] findet man experimen-

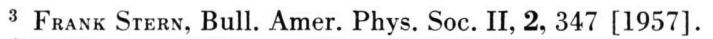

4 D. Geist u. G. Römelt, Phys. Verh. 9, 30 [1958].

\section{Zur Zeitkonstanten der Sekundäremission}

Von H. W. Streitwolf und W. Brauer

Institut für Festkörperforschung der Deutschen Akademie der Wissenschaften zu Berlin

(Z. Naturforschg. 13 a, 700-701 [1958] ; eingegangen am 12. Mai 1958)

Die Einstellzeit $\tau$ der Sekundäremission ist die Zeit, die zwischen dem Eindringen des Primärstrahls in den Emitter und dem Erreichen des stationären Ausbeutewertes vergeht. Prinzipiell wäre diese Zeit zu berechnen aus dem zeitlichen Verhalten der Lösung der nichtstationären Boltzmannschen Transportgleichung für die Sekundärelektronen (S) an der Oberfläche des Emitters. Nach vaN DER $Z_{\text {IEL }}{ }^{1}$ kann man aber $\tau$ auf Grund einer einfachen energetischen Betrachtung abschätzen. Es gilt nämlich die Energiebilanz

$$
j_{\mathrm{p}} E_{\mathrm{p}} \tau=\frac{1}{\delta} j_{\mathrm{s}} E_{\mathrm{p}} \tau \gtrsim N E_{\mathrm{e}}
$$

( $j_{\mathrm{p}}$ bzw. $j_{\mathrm{s}}$ primäre bzw. sekundäre Stromdichte, $E_{\mathrm{p}}$ Primärenergie, $N$ mittlere Anzahl angeregter $\mathrm{S}$ pro $\mathrm{cm}^{2}$ Oberfläche, $E_{\mathrm{e}}$ mittlere Anregungsenergie, $\delta$ Ausbeute).

In der Nähe des Maximums $E_{\mathrm{pm}}$ setzen wir $N=n d_{\mathrm{s}}$ ( $n$ Dichte der inneren $\mathrm{S}$ an der Oberfläche, $d_{\mathrm{s}}$ Austrittstiefe der S). $n, E_{\mathrm{e}}$ und $j_{\mathrm{s}}$ ergeben sich aus der EnergieWinkel-Verteilung der inneren $\mathrm{S}$ durch Integration. $\mathrm{V}_{\text {AN }}$ DER Z Z ${ }_{\text {IEL }}{ }^{1}$ benutzt eine MAXwelL-Verteilung mit einer formalen Temperatur entsprechend $2-3 \mathrm{eV}$. Die äußere Verteilung der S verläuft bekanntlich in guter Näherung nach einem solchen Gesetz. Da aber tatsächlich die innere Verteilung benötigt wird, erscheint der VAN DER ZIELsche Ansatz in dieser Hinsicht nicht gerechtfertigt und soll im folgenden durch genauere Betrachtungen ersetzt werden.

Mit dem Wechselwirkungspotential

$$
H_{\mathrm{W}}=e^{2} / r \cdot \exp \{-\lambda r\}, \quad \lambda=10^{8} \mathrm{~cm}^{-1}
$$

erhält man allgemein für die im $\mathrm{cm}^{3}$ pro sec angereg. ten $\mathrm{S}$ bezogen auf die Primärstromdichte Eins

$$
S_{\lambda}(E)=\frac{4 m e^{4}}{V \hbar^{2} K} k^{\prime 2} \frac{\mathrm{d} k^{\prime}}{\mathrm{d} E} \sum_{\mathfrak{S}} \sum_{\mathfrak{\mathrm { f }}} \int \mathrm{d} \Omega_{\mathrm{f}^{\prime}} \frac{|I|^{2}}{\left(q^{2}+\lambda^{2}\right)^{2}} \delta\left(E_{\mathrm{f}^{\prime} \Omega^{\prime}}-E_{\mathfrak{f} \Omega}\right)
$$

tell ${ }^{4}$ (durchgezogene Kurve) über $10^{18} \mathrm{~cm}^{-3}$ Elektronen ein Absinken der Elektronensuszeptibilität. Dieses Verhalten wird durch die mit den Werten von STERN berechnete Kurve beschrieben (strichpunktiert). Der berechnete Bandverlauf dürfte demnach zumindest in groBen Zügen zutreffen.

Herrn Prof. Dr. J. Jaumann danke ich für die Möglichkeit zur Durchführung dieser Untersuchungen. Mit Herrn G. Römelt, Köln, führte ich anregende Diskussionen; Herrn R. Bowers, Pittsburgh, danke ich sehr für die spontane Mitteilung der Berechnungen von F. STERN.

mit der Nebenbedingung

$$
\mathfrak{R}^{\prime}=\mathfrak{R}+\mathfrak{k}+2 \pi \mathfrak{H}-\mathfrak{k}^{\prime} .
$$

Dabei ist $E=\left(\hbar^{2} / 2 m\right) k^{\prime 2}$ die Energie des angeregten $S$, $\hbar \mathfrak{q}=\hbar\left(\Omega-\Omega^{\prime}\right)$ der übertragene Impuls, $\mathfrak{H}$ ein Vektor aus dem reziproken Gitter und $V$ das Normierungsvolumen. Der Formfaktor $I$ hängt in unserem Falle nicht von den Endzuständen ab und kann vor das Integral gezogen werden. Wir berücksichtigen nur das Summenglied mit $\mathfrak{H}=0$.

Nehmen wir für Isolatoren ein unendlich schmales Valenzband an und beschreiben die Valenzelektronen alle durch die Buochsche Welle $\psi_{0}=(1 / \sqrt{V}) u_{0}(\mathrm{r}), \mathfrak{k}=0$, so wird die Anregungsfunktion (wir setzen $\lambda=0$ )

$$
S(E)= \begin{cases}0 & \text { für } \quad E<\frac{(\Delta E)^{2}}{4 E_{\mathrm{p}}}, \\ \frac{D_{0} e^{4} \mid I !^{2} \pi}{E_{\mathrm{p}} E^{2}} & \text { für } \quad E>\frac{(\Delta E)^{2}}{4 E_{\mathrm{p}}} .\end{cases}
$$

Dabei ist $D_{0}$ die Valenzelektronendichte und $\Delta E$ die Breite der verbotenen Zone.

Bei der Diffusion der S zur Oberfläche berücksichtigen wir nur ihre Wechselwirkung mit den Phononen mit Hilfe der Age-Gleichung (DeKKer ${ }^{2}$ ).

Nach Deккеr erhält man als Lösung der Age-Gleichung

$$
n_{\mathrm{E}}(E)-n_{\mathrm{E}}(\Delta E)=\gamma \sqrt{E} \int_{E}^{\Delta E} S\left(E^{\prime}\right) \mathrm{d} E^{\prime} .
$$

$\gamma$ ist eine hier unwesentliche Konstante. Wir vernachlässigen die wenigen schnellen $\mathrm{S}$ und setzen

$$
n_{\mathrm{E}}(\Delta E)=0 \text {. }
$$

Mit der Anregungsfunktion (3) erhält man

$$
\begin{aligned}
n_{\mathrm{E}}(E) & =\frac{\gamma D_{0} e^{4}|I|^{2} \pi}{E_{\mathrm{p}}} \\
& \cdot \sqrt{E} \cdot\left\{\begin{array}{lll}
\frac{1}{E}-\frac{1}{\Delta E} & \text { für } & \frac{(\Delta E)^{2}}{4 E_{\mathrm{p}}}<E<\Delta E, \\
\frac{4 E_{\mathrm{p}}}{(\Delta E)^{2}}-\frac{1}{\Delta E} & \text { für } & E<\frac{(\Delta E)^{2}}{4 E_{\mathrm{p}}} .
\end{array}\right.
\end{aligned}
$$

1 A. VAN der ZieL, J. Appl. Phys. 28, 1216 [1957].

2 A. J. Deкker, Physica 21, 29 [1954]. 